DESY 09-204

WU B 09-17

\title{
Two-photon annihilation into octet meson pairs: symmetry relations in the handbag approach
}

\author{
M. Diehl \\ Deutsches Elektronen-Synchroton DESY, 22603 Hamburg, Germany \\ P. Kroll \\ Fachbereich Physik, Universität Wuppertal, 42097 Wuppertal, Germany \\ and \\ Institut für Theoretische Physik, Universität Regensburg, 93040 Regensburg, Germany
}

\begin{abstract}
We explore the implications of SU(3) flavor symmetry in the soft handbag mechanism for two-photon annihilation into pairs of pseudoscalar octet mesons. In this approach we obtain a good description of the experimental results for all measured channels at high energy, with two complex form factors adjusted to the data. We also predict the cross section for $\gamma \gamma \rightarrow \eta \eta$.
\end{abstract}




\section{Introduction}

A few years ago, we have investigated two-photon annihilation into pairs of pseudoscalar mesons within the soft handbag approach [1]. The physical mechanism described by this approach is a parton-level process $\gamma \gamma \rightarrow q \bar{q}$ followed by hadronization of the $q \bar{q}$ pair into the final-state mesons, where each meson approximately carries the momentum of the quark or antiquark. To ensure that the parton-level process proceeds at short distances, one must require large Mandelstam variables $s, t$, and $u$. Neglecting contributions that are suppressed in this kinematics, we have derived the cross section formula

$$
\frac{d \sigma}{d t}(\gamma \gamma \rightarrow M \bar{M})=\frac{8 \pi \alpha_{\mathrm{em}}^{2}}{s^{2}} \frac{1}{\sin ^{4} \theta}\left|R_{M \bar{M}}(s)\right|^{2},
$$

where $\theta$ is the c.m. scattering angle. The form factor $R_{M} \bar{M}(s)$ describes the soft transition from $q \bar{q}$ to the meson pair. It can be written as the matrix element of the quark part of the energy-momentum tensor, summed over quark flavors with appropriate charge factors and taken between the $M \bar{M}$ state and the vacuum. A key prediction of the handbag approach is the absence of final states with isospin $I=2$, given that the process proceeds through a single intermediate $q \bar{q}$ pair. Using approximate flavor $\mathrm{SU}(3)$ symmetry for the transition form factors one obtains a number of further predictions, which are discussed in detail in the present work.

The soft handbag mechanism for $\gamma \gamma$ annihilation into a hadron pair has a spacelike analog in Compton scattering at large Mandelstam variables [2], and its dynamics is closely related with the Feynman mechanism for form factors at large momentum transfer. As in the latter case, one can show that at asymptotically large momenta another mechanism will be dominant, namely the hard-scattering mechanism of Brodsky and Lepage [3]. For the process under discussion, the amplitude then factorizes into a hard partonic subprocess $\gamma \gamma \rightarrow q \bar{q} q \bar{q}$ and a single-meson distribution amplitude for each meson [4]. Since there are two $q \bar{q}$ pairs in the intermediate state, $I=2$ transitions are allowed, in contrast to the handbag approach. Which mechanism is relevant in given kinematics can of course not be determined by general arguments, and one of the purposes of this paper is a detailed comparison of soft handbag results with data.

In the past years, the BELLE collaboration has published data on exclusive $\gamma \gamma$ annihilation for many different pseudoscalar meson channels [5, 6, 7, 8]. These data are significantly more accurate and cover more final states than previous measurements [9, 10, which were only available for $\pi^{+} \pi^{-}$and $K^{+} K^{-}$pairs. In view of this situation we find it timely to update our initial analysis in [1]. The BELLE data [5, 6, 7, 8] nicely confirm the $1 / \sin ^{4} \theta$ behavior (1) of the differential cross sections for $s$ larger than about $9 \mathrm{GeV}^{2}$. This allows us to determine the process form factors from cross sections integrated over a given interval of $\theta$ around $90^{\circ}$, where the handbag approach is applicable. The cross section integrated over $\cos \theta$ from $-\cos \theta_{0}$ to $\cos \theta_{0}$ reads

$$
\sigma(\gamma \gamma \rightarrow M \bar{M})=S \frac{4 \pi \alpha_{\mathrm{em}}^{2}}{s}\left[\frac{\cos \theta_{0}}{\sin ^{2} \theta_{0}}+\frac{1}{2} \ln \frac{1+\cos \theta_{0}}{1-\cos \theta_{0}}\right]\left|R_{M \bar{M}}(s)\right|^{2},
$$

where the statistical factor $S$ is equal to $1 / 2$ for two identical mesons like $\pi^{0} \pi^{0}$ and equal to unity otherwise. Most of the experimental data for the integrated cross section are quoted for $\cos \theta_{0}=0.6$. An exception is the $\eta \pi^{0}$ cross section in [8, which is given for $\cos \theta_{0}=0.8$. The 
numerical constants that give the cross section (2) when multiplied with $s^{-1}\left|R_{M \bar{M}}(s)\right|^{2}$ are $425 \mathrm{nb} \mathrm{GeV}^{2}$ for $\cos \theta_{0}=0.6$ and $866 \mathrm{nb} \mathrm{GeV}^{2}$ for $\cos \theta_{0}=0.8$ in the case where $S=1$.

The plan of this paper is as follows. In the next section we discuss the flavor-symmetry properties of the handbag amplitude, with particular emphasis on the $\eta \pi^{0}$ and $\eta \eta$ channels, which have not been considered before and add to the predictive power of the approach. In Sect. 3 we extract the annihilation form factor $R_{M \bar{M}}$ for each of the available meson channels, while in Sect. 4 we analyze the annihilation form factors in terms of the two independent quarklevel form factors, a valence and a non-valence one. In Sect. 5 we point out some differences between the handbag approach and the hard-scattering mechanism of Brodsky and Lepage, before summarizing our main results in Sect. 6.

\section{Flavor symmetry relations in the handbag approach}

To derive flavor SU(3) relations between the annihilation amplitudes into different meson pairs, we find it convenient to consider the usual isospin multiplets and in addition the multiplets under $U$-spin and $V$-spin, which are the $\mathrm{SU}(2)$ symmetries associated with the exchange $s \leftrightarrow d$ and $u \leftrightarrow s$, respectively [11]. The corresponding singlets, doublets, and triplets read

$$
\begin{array}{llll}
U: & \frac{1}{2}\left(\eta+\sqrt{3} \pi^{0}\right), & \left\{\pi^{+}, K^{+}\right\}, & \left\{\bar{K}^{0}, \frac{1}{2}\left(\sqrt{3} \eta-\pi^{0}\right), K^{0}\right\}, \\
V: & \frac{1}{2}\left(\eta-\sqrt{3} \pi^{0}\right), & \left\{K^{0}, \pi^{-}\right\}, & \left\{K^{+}, \frac{1}{2}\left(\sqrt{3} \eta+\pi^{0}\right), K^{-}\right\},
\end{array}
$$

where our phase conventions for meson states correspond to the quark content

$$
\begin{aligned}
& \pi^{+}=\bar{d} u, \quad \pi^{-}=\bar{u} d, \quad \pi^{0}=\frac{1}{\sqrt{2}}(\bar{u} u-\bar{d} d), \\
& K^{+}=\bar{s} u, \quad K^{-}=\bar{u} s, \\
& K^{0}=\bar{s} d, \quad \bar{K}^{0}=\bar{d} s, \quad \eta=\frac{1}{\sqrt{6}}(\bar{u} u+\bar{d} d-2 \bar{s} s) .
\end{aligned}
$$

At this stage we neglect $\eta-\eta^{\prime}$ mixing and approximate the physical $\eta$ as a pure flavor octet state. In the process amplitudes and annihilation form factors for $\gamma \gamma \rightarrow M_{1} M_{2}$, the two-meson states appear in the $C$-even combinations

$$
\left|M_{1} M_{2}\right\rangle=\frac{\left|M_{1}(p) M_{2}\left(p^{\prime}\right)\right\rangle+\left|M_{1}\left(p^{\prime}\right) M_{2}(p)\right\rangle}{2},
$$

so that $\left|M_{1} M_{2}\right\rangle=\left|M_{2} M_{1}\right\rangle$. From these two-meson states we can form linear combinations $\left|\Phi_{I}\right\rangle,\left|\Phi_{U}\right\rangle,\left|\Phi_{V}\right\rangle$ with definite isospin, $U$-spin, and $V$-spin, respectively.

$U$-spin symmetry plays a special role in our context, because the photon is a $U$-spin singlet. From the absence of two-photon transitions to the states $\Phi_{U=1}$ and $\Phi_{U=2}$ we immediately obtain the following relations for the amplitudes of $\gamma \gamma \rightarrow M_{1} M_{2}$ :

$$
\begin{aligned}
\mathcal{A}_{K^{+} K^{-}} & =\mathcal{A}_{\pi^{+} \pi^{-}}, \\
\mathcal{A}_{K^{0} \bar{K}^{0}} & =\frac{3}{4} \mathcal{A}_{\eta \eta}+\frac{1}{4} \mathcal{A}_{\pi^{0} \pi^{0}}-\frac{\sqrt{3}}{2} \mathcal{A}_{\eta \pi^{0}} .
\end{aligned}
$$

These relations are respected by any dynamical mechanism in the limit of SU(3) symmetry. 
A further set of relations emerges in the soft handbag mechanism because the two photons annihilate via a quark-antiquark intermediate state. This allows only final states with $I$ and $V$ equal to 0 or 1 . The absence of $I=2$ and $V=2$ states respectively implies

$$
\begin{aligned}
\mathcal{A}_{\pi^{0} \pi^{0}} & =\mathcal{A}_{\pi^{+} \pi^{-}}, \\
\mathcal{A}_{K^{+} K^{-}} & =\frac{3}{4} \mathcal{A}_{\eta \eta}+\frac{1}{4} \mathcal{A}_{\pi^{0} \pi^{0}}+\frac{\sqrt{3}}{2} \mathcal{A}_{\eta \pi^{0}} .
\end{aligned}
$$

In the $\mathrm{SU}(3)$ limit, the handbag mechanism thus predicts equal differential cross sections for the channels $K^{+} K^{-}, \pi^{+} \pi^{-}$, and $\pi^{0} \pi^{0}$, as was already pointed out in [1]. With this equality, the sum and the difference of (7) and (9) give relations between only three different channels:

$$
\begin{aligned}
\mathcal{A}_{\eta \pi^{0}} & =\frac{1}{\sqrt{3}}\left(\mathcal{A}_{K^{+} K^{-}}-\mathcal{A}_{K^{0} \bar{K}^{0}}\right), \\
\mathcal{A}_{\eta \eta} & =\frac{1}{3} \mathcal{A}_{K^{+} K^{-}}+\frac{2}{3} \mathcal{A}_{K^{0} \bar{K}^{0}},
\end{aligned}
$$

or equivalently

$$
\begin{aligned}
\mathcal{A}_{K^{+} K^{-}} & =\mathcal{A}_{\eta \eta}+\frac{2}{\sqrt{3}} \mathcal{A}_{\eta \pi^{0}}, \\
\mathcal{A}_{K^{0} \bar{K}^{0}} & =\mathcal{A}_{\eta \eta}-\frac{1}{\sqrt{3}} \mathcal{A}_{\eta \pi^{0}} .
\end{aligned}
$$

Let us work out the consequences of (10) to (13), which involve the final states $K^{+} K^{-}, K^{0} \bar{K}^{0}$, $\eta \pi^{0}$, and $\eta \eta$. The square of each relation contains a term depending on the relative phase between the two amplitudes on the right-hand side. This yields triangular inequalities between any combination of three cross sections. The first of these reads

$$
\left|\sqrt{\frac{1}{3} \frac{d \sigma_{K^{+} K^{-}}}{d t}}-\sqrt{\frac{1}{3} \frac{d \sigma_{K^{0} \bar{K}^{0}}}{d t}}\right| \leq \sqrt{\frac{d \sigma_{\eta \pi^{0}}}{d t}} \leq \sqrt{\frac{1}{3} \frac{d \sigma_{K^{+} K^{-}}}{d t}}+\sqrt{\frac{1}{3} \frac{d \sigma_{K^{0} \bar{K}^{0}}}{d t}}
$$

and the others are readily obtained in analogy. One obtains one relation between all four cross sections in which phases between amplitudes drop out:

$$
2 \frac{d \sigma_{\eta \pi^{0}}}{d t}+3 \frac{d \sigma_{\eta \eta}}{d t}=\frac{d \sigma_{K^{+} K^{-}}}{d t}+2 \frac{d \sigma_{K^{0} \bar{K}^{0}}}{d t}
$$

which may be regarded as the $\mathrm{SU}(3)$ analog of the isospin relation $d \sigma_{\pi^{0} \pi^{0}} / d t=d \sigma_{\pi^{+} \pi^{-}} / d t$.

Having derived relations between the full process amplitudes in the handbag mechanism, we now turn our attention to the annihilation form factors for each separate quark flavor. $\mathrm{SU}(3)$ flavor symmetry gives

$$
\begin{array}{lll}
R_{\Phi_{I=0}}^{u}=R_{\Phi_{I=0}}^{d}, & R_{\Phi_{I=1}}^{u}=-R_{\Phi_{I=1}}^{d}, & R_{\Phi_{I=1}}^{s}=R_{\Phi_{I=2}}^{s}=R_{\Phi_{I=2}}^{u}=R_{\Phi_{I=2}}^{d}=0, \\
R_{\Phi_{U=0}}^{s}=R_{\Phi_{U=0}}^{d}, & R_{\Phi_{U=1}}^{s}=-R_{\Phi_{U=1}}^{d}, & R_{\Phi_{U=1}}^{u}=R_{\Phi_{U=2}}^{u}=R_{\Phi_{U=2}}^{s}=R_{\Phi_{U=2}}^{d}=0
\end{array}
$$

for the annihilation form factors into states with definite isospin or $U$-spin. With this we can express all annihilation form factors in terms of a valence form factor

$$
\begin{aligned}
R_{2 \pi}^{u} & \stackrel{\text { def }}{=} R_{\pi^{+} \pi^{-}}^{u}=R_{\pi^{+} \pi^{-}}^{d}=R_{\pi^{0} \pi^{0}}^{u}=R_{\pi^{0} \pi^{0}}^{d} \\
& =R_{K^{+} K^{-}}^{u}=R_{K^{+} K^{-}}^{s}=R_{K^{0} \bar{K}^{0}}^{d}=R_{K^{0} \bar{K}^{0}}^{s}
\end{aligned}
$$


and a non-valence form factor

$$
R_{2 \pi}^{s} \stackrel{\text { def }}{=} R_{\pi^{+} \pi^{-}}^{s}=R_{\pi^{0} \pi^{0}}^{s}=R_{K^{+} K^{-}}^{d}=R_{K^{0} \bar{K}^{0}}^{u}
$$

These results were already given in [1]. In addition we obtain

$$
\begin{aligned}
R_{\eta \pi^{0}}^{u} & =-R_{\eta \pi^{0}}^{d}=\frac{1}{\sqrt{3}}\left(R_{2 \pi}^{u}-R_{2 \pi}^{s}\right), & R_{\eta \pi^{0}}^{s} & =0, \\
R_{\eta \eta}^{u} & =R_{\eta \eta}^{d}=\frac{1}{3} R_{2 \pi}^{u}+\frac{2}{3} R_{2 \pi}^{s}, & R_{\eta \eta}^{s} & =\frac{4}{3} R_{2 \pi}^{u}-\frac{1}{3} R_{2 \pi}^{s} .
\end{aligned}
$$

It is instructive to compare these results with the corresponding flavor $\mathrm{SU}(3)$ relations for the production of $B \bar{B}$ pairs, where $B$ is a member of the ground state baryon octet [12]. In the meson case there are two independent form factors, whereas in the baryon case there are three. This is because there are fewer meson-antimeson states, given that the antimeson octet is equal to the meson octet. In the baryon case we have for instance two distinct states $\left|\Sigma^{+} \bar{\Sigma}^{-}\right\rangle$and $\left|\Sigma^{-} \bar{\Sigma}^{+}\right\rangle$, whereas the corresponding meson states $\left|\pi^{+} \pi^{-}\right\rangle$and $\left|\pi^{-} \pi^{+}\right\rangle$are equivalent according to (5). Taking this into account, the relations (17) to (19) are the analog of eq. (40) in [12].

Summing up the individual quark flavor contributions to each channel as

$$
R_{M \bar{M}}=\frac{4}{9} R_{M \bar{M}}^{u}+\frac{1}{9} R_{M \bar{M}}^{d}+\frac{1}{9} R_{M \bar{M}}^{s}
$$

we find that (17) to (19) implies

$$
R_{\pi^{0} \pi^{0}}=R_{\pi^{+} \pi^{-}}=R_{K^{+} K^{-}}
$$

and

$$
\begin{aligned}
R_{\pi^{+} \pi^{-}} & =\frac{5}{9} R_{2 \pi}^{u}+\frac{1}{9} R_{2 \pi}^{s}, \\
R_{K^{0} \bar{K}^{0}} & =\frac{2}{9} R_{2 \pi}^{u}+\frac{4}{9} R_{2 \pi}^{s}, \\
R_{\eta \pi^{0}} & =\frac{1}{3 \sqrt{3}}\left(R_{2 \pi}^{u}-R_{2 \pi}^{s}\right), \\
R_{\eta \eta} & =\frac{1}{3}\left(R_{2 \pi}^{u}+R_{2 \pi}^{s}\right) .
\end{aligned}
$$

Only the equality $R_{\pi^{+} \pi^{-}}=R_{\pi^{0} \pi^{0}}$ and the first relation in (22) follow from isospin symmetry alone, whereas all other relations need full SU(3) flavor symmetry. From (22) one can readily rederive the relations (10) to (13) for the process amplitudes.

If one assumes that the soft handbag mechanism is valid within a certain accuracy, one can use the cross sections for any three of the channels $K^{+} K^{-}, K^{0} \bar{K}^{0}, \eta \pi^{0}, \eta \eta$ to extract the size of the form factors $R_{2 \pi}^{u}$ and $R_{2 \pi}^{s}$ as well as their relative phase $\rho$. Taking the squares of the first three relations in (22) and forming appropriate linear combinations, we obtain for instance

$$
\begin{array}{r}
f(s, \theta)\left|R_{2 \pi}^{u}\right|^{2}=-\frac{d \sigma_{K^{0} \bar{K}^{0}}}{d t}+4 \frac{d \sigma_{\eta \pi^{0}}}{d t}+4 \frac{d \sigma_{K^{+} K^{-}}}{d t}, \\
f(s, \theta)\left|R_{2 \pi}^{s}\right|^{2}=5 \frac{d \sigma_{K^{0} \bar{K}^{0}}}{d t}+10 \frac{d \sigma_{\eta \pi^{0}}}{d t}-2 \frac{d \sigma_{K^{+} K^{-}}}{d t}, \\
f(s, \theta)\left|R_{2 \pi}^{u} R_{2 \pi}^{s}\right| \cos \rho=2 \frac{d \sigma_{K^{0} \bar{K}^{0}}}{d t}-11 \frac{d \sigma_{\eta \pi^{0}}}{d t}+\frac{d \sigma_{K^{+} K^{-}}}{d t},
\end{array}
$$

where

$$
f(s, \theta)=\frac{32 \pi \alpha_{\mathrm{em}}^{2}}{3 s^{2} \sin ^{4} \theta} .
$$




\section{Extraction of the process form factors}

The amplitudes for the production of pion pairs in two-photon annihilation have the isospin decomposition

$$
\begin{aligned}
\mathcal{A}_{\pi^{+} \pi^{-}} & =\frac{1}{\sqrt{3}} \mathcal{A}_{2 \pi}^{I=0}+\frac{1}{\sqrt{6}} \mathcal{A}_{2 \pi}^{I=2}, \\
\mathcal{A}_{\pi^{0} \pi^{0}} & =\frac{1}{\sqrt{3}} \mathcal{A}_{2 \pi}^{I=0}-\frac{2}{\sqrt{6}} \mathcal{A}_{2 \pi}^{I=2},
\end{aligned}
$$

with the $I=1$ final state being forbidden by charge conjugation. The absence of $I=2$ transitions, which is a central property of the handbag approach as discussed above, gives

$$
R \stackrel{\text { def }}{=} \frac{\sigma\left(\gamma \gamma \rightarrow \pi^{0} \pi^{0}\right)}{\sigma\left(\gamma \gamma \rightarrow \pi^{+} \pi^{-}\right)}=\frac{1}{2}
$$

for the ratio of integrated cross sections, where the statistical factor $S=1 / 2$ is taken into account for the $\pi^{0} \pi^{0}$ channel. By contrast, one has $R=2$ if $I=0$ transitions are absent. As shown in Fig. 1, the experimental value of $R$ is about 0.3 at $s=9 \mathrm{GeV}^{2}$ and increases for higher energies, with central values between 0.3 and 0.5 and rapidly growing errors.

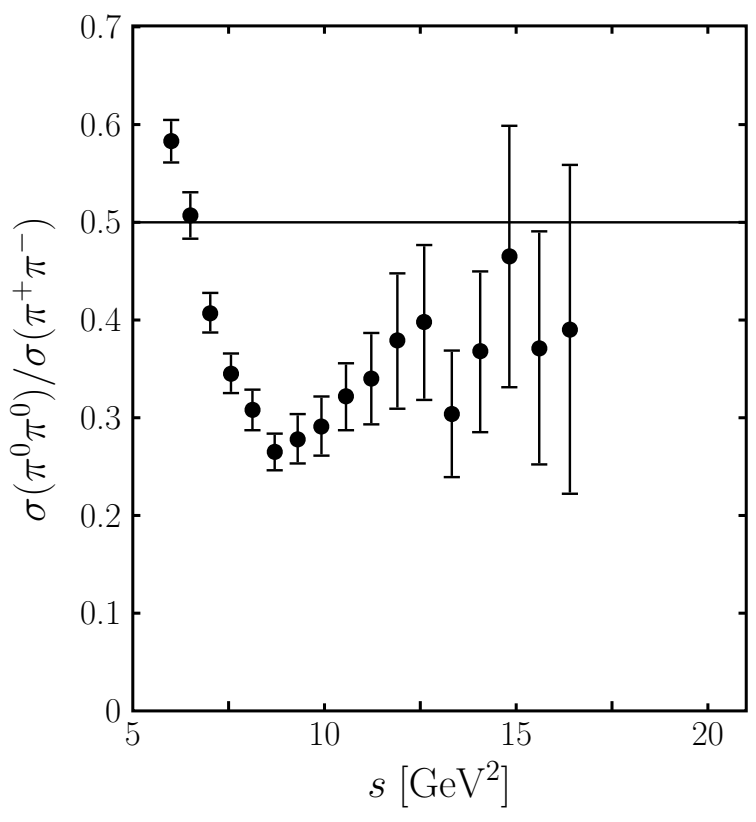

Figure 1: BELLE data [6] for the cross section ratio of neutral and charged pion pair production. Contributions from charmonium decay have been subtracted by BELLE. The errors shown are statistical only.

Because amplitudes enter quadratically in cross sections, one cannot uniquely reconstruct the ratio $r=\mathcal{A}_{2 \pi}^{I=2} / \mathcal{A}_{2 \pi}^{I=0}$ of isospin amplitudes from the cross section ratio $R$, even for a given relative phase between $\mathcal{A}_{2 \pi}^{I=0}$ and $\mathcal{A}_{2 \pi}^{I=2}$. For instance, both $r=0$ and $r=2 \sqrt{2}$ give $R=1 / 2$, and both $r=\sqrt{2} / 14 \approx 0.1$ and $r=3 / \sqrt{2} \approx 2.1$ give $R=8 / 25=0.32$. If one assumes that the two amplitudes are in phase and that $\mathcal{A}_{2 \pi}^{I=2}<\mathcal{A}_{2 \pi}^{I=0}$, then $0.3<R<0.5$ implies 

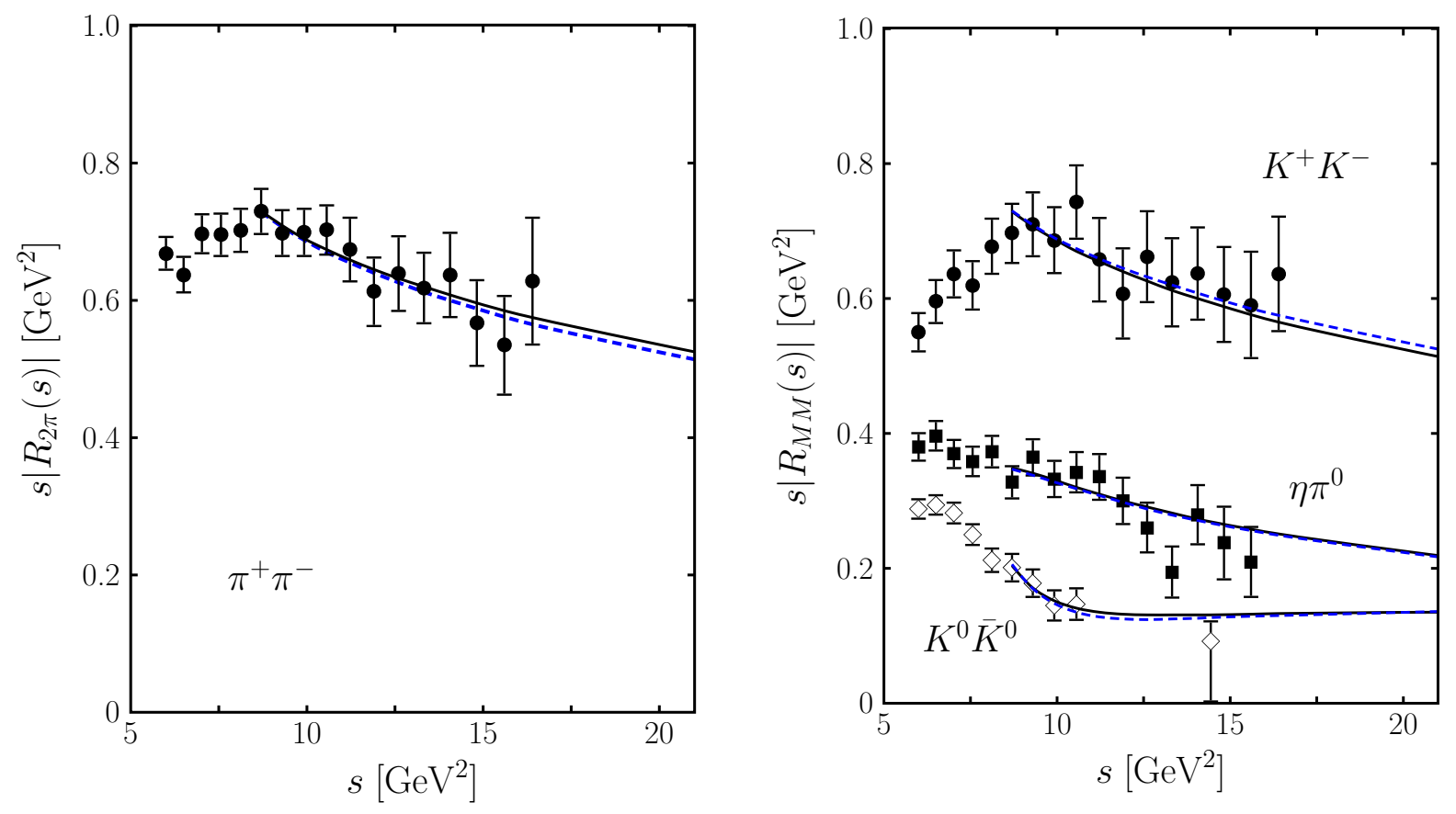

Figure 2: Left: The modulus of the scaled annihilation form factor $s R_{\pi \pi}(s)$, extracted from the combination (27) of $\pi^{0} \pi^{0}$ and $\pi^{+} \pi^{-}$cross sections. Data are taken from [5, 6]. Right: The scaled annihilation form factors for the $K^{+} K^{-}, K^{0} \bar{K}^{0}$, and $\eta \pi^{0}$ channels. Data are from [5] for $K^{+} K^{-}$(filled circles), from [8] for $\eta \pi^{0}$ (filled squares), and from [7] for $K^{0} \bar{K}^{0}$ (open diamonds). Here and in all subsequent plots, the error bars combine statistical and systematic uncertainties in quadrature, and solid (dashed) lines represent fit 1 (2) specified in Tab. 2 .

$\mathcal{A}_{2 \pi}^{I=2} / \mathcal{A}_{2 \pi}^{I=0}<0.11$. We thus see that the data on $\pi^{0} \pi^{0}$ and $\pi^{+} \pi^{-}$production for $s>9 \mathrm{GeV}^{2}$ cannot prove the dominance of $I=0$ transitions but is fully consistent with it.

With this situation in mind we determine the process form factor $R_{\pi \pi}$ from the combination

$$
\frac{2}{3}\left[\sigma\left(\gamma \gamma \rightarrow \pi^{0} \pi^{0}\right)+\sigma\left(\gamma \gamma \rightarrow \pi^{+} \pi^{-}\right)\right],
$$

where the interference term between the $I=0$ and $I=2$ transitions cancels and where the squared amplitudes $\left|\mathcal{A}_{2 \pi}^{I=0}\right|^{2}$ and $\left|\mathcal{A}_{2 \pi}^{I=2}\right|^{2}$ enter with equal weight. The form factor obtained from this combination is almost pure $I=0$ and only mildly contaminated by the $I=2$ contribution, provided that $\left|\mathcal{A}_{2 \pi}^{I=2} / \mathcal{A}_{2 \pi}^{I=0}\right|$ is small compared to 1 , which we assume from now on. The results for $R_{\pi \pi}(s)$ obtained from the BELLE data [5, 6] are shown in the left panel of Fig. 2. Here and in the following we add statistical and systematic errors of the data in quadrature.

We proceed by extracting the process form factors for the $K^{+} K^{-}, K^{0} \bar{K}^{0}$, and $\eta \pi^{0}$ channels from the corresponding cross sections measured by the BELLE collaboration [5, 7, 8]. Note that BELLE measured the cross section for the production of $K$-short pairs, which is related to the $K^{0} \bar{K}^{0}$ cross section by

$$
\sigma\left(\gamma \gamma \rightarrow K^{0} \bar{K}^{0}\right)=2 \sigma\left(\gamma \gamma \rightarrow K_{S} K_{S}\right) .
$$


The c.m. energies we consider in our analysis include the charmonium region. For $\pi^{0} \pi^{0}, \pi^{+} \pi^{-}$, and $K^{+} K^{-}$the BELLE collaboration corrected their data for contributions from the $\chi_{c J}$ states. A corresponding subtraction was not performed for the $K_{S} K_{S}$ channel, where instead no cross section was given for $10.5 \mathrm{GeV}^{2}<s<14.4 \mathrm{GeV}^{2}$. Note that $\chi_{c J}$ states do not contribute to $\eta \pi^{0}$ production. The cross sections we calculate in the handbag approach are of course to be understood as excluding contributions from charmonium decays.

The extracted form factors are shown in the right panel of Fig. 2, As in the case of pion pairs, the $s$ dependence of the form factors is somewhat steeper than the $1 / s$ behavior predicted by dimensional counting. In particular, the $K^{0} \bar{K}^{0}$ form factor decreases sharply with energy. Such a behavior is not in conflict with the soft handbag approach, given that the dimensional counting rules reflect the dynamics of Brodsky-Lepage hard scattering rather than the dynamics of the Feynman mechanism. What the soft handbag approach does predict is that the non-valence form factor $R_{2 \pi}^{s}$ should fall off more rapidly with $s$ than its valence counterpart $R_{2 \pi}^{u}$. This is because the soft mechanism requires that the momentum fraction of a final-state meson with respect to the initial quark or antiquark must approach unity as $s$ increases [1]. In a pion this is of course more likely to happen for $u \bar{u}$ than for $s \bar{s}$.

For $s \gtrsim 8 \mathrm{GeV}^{2}$ the $K^{0} \bar{K}^{0}$ form factor is smaller than predicted by the relation

$$
R_{K^{0} \bar{K}^{0}} \simeq \frac{2}{5} R_{K^{+} K^{-}}
$$

which is obtained from the $\mathrm{SU}(3)$ symmetry constraints (21) and (22) if the non-valence form factor $R_{2 \pi}^{s}$ is negligible. Thus, the behavior of $R_{K^{0} \bar{K}^{0}}$ clearly necessitates contributions from the non-valence form factor at presently available energies. The relation (29) is then expected to hold only for very large $s$.

As quoted in [5] the $U$-spin relation (6) between the $\pi^{+} \pi^{-}$and $K^{+} K^{-}$amplitudes (or form factors) is fairly well respected by the data. The ratio of $K^{+} K^{-}$and $\pi^{+} \pi^{-}$cross sections is $0.89 \pm 0.04$ (stat.) \pm 0.15 (syst.) for $s>9 \mathrm{GeV}^{2}$ [5]. When we use the combination (27) to extract $R_{\pi \pi}$ the agreement with $R_{K^{+} K^{-}}$is even better, as shown in the left panel of Fig. 3, For $s \gtrsim 8 \mathrm{GeV}^{2}$ this ratio is perfectly compatible with unity. Even the errors on the ratio are not unreasonably large.

As another test of the handbag approach combined with flavor symmetry, we may verify the bounds (14) on the $\eta \pi^{0}$ cross section, or equivalently on the corresponding form factor $R_{\eta \pi^{0}}$. As is evident from Fig. 3, this form factor is much smaller than the upper bound

$$
\frac{\left|R_{K^{+} K^{-}}\right|+\left|R_{K^{0} \bar{K}^{0}}\right|}{\sqrt{3}}
$$

but rather close to the lower bound

$$
\frac{\left|R_{K^{+} K^{-}}\right|-\left|R_{K^{0} \bar{K}^{0}}\right|}{\sqrt{3}} .
$$

For the first three energies above $s=9 \mathrm{GeV}^{2}$, the lower bound is well respected by the $\eta \pi^{0}$ data. For $s=10.92 \mathrm{GeV}^{2}$ the bound is practically saturated and for $s=14.44 \mathrm{GeV}^{2}$ it is slightly violated if one considers the central values of the cross sections. Within the experimental errors, the lower bound is, however, consistent with the data also at the highest energy. 

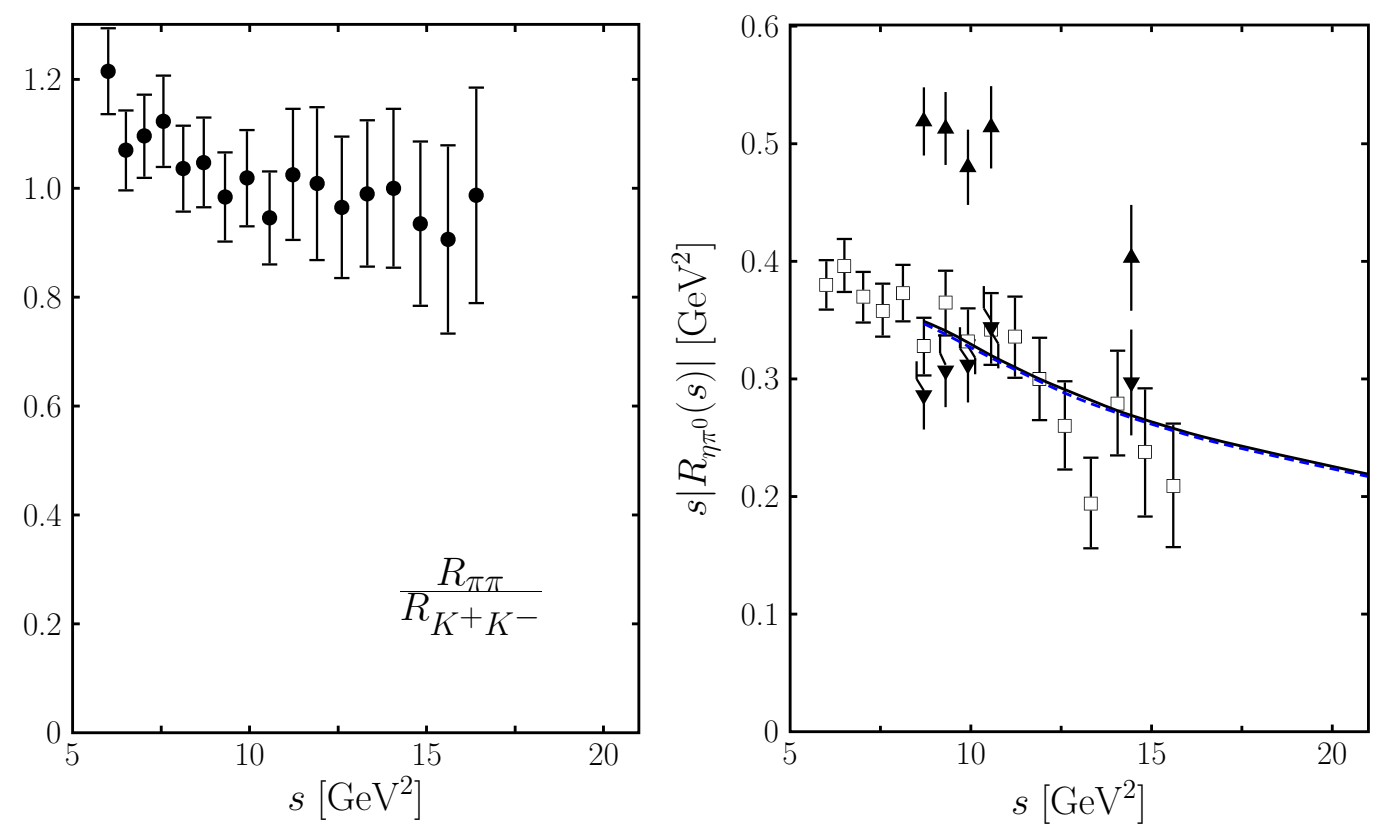

Figure 3: Left: The ratio of $\pi \pi$ and $K^{+} K^{-}$annihilation form factors, where $R_{\pi \pi}$ is obtained from the cross section combination (27). Right: Probing the bounds (30) and (31) for the $\eta \pi^{0}$ form factor. Open squares represent the scaled $\eta \pi^{0}$ form factor extracted from the data of [8], whereas (reversed) triangles represent the upper (lower) bound evaluated from the data given in [5, 7]. The solid and dashed curves have the same meaning as in Fig. 2,

\section{Combined analysis of meson octet channels}

Having investigated the annihilation form factors for the individual meson channels, we now determine the basic valence and non-valence form factors $R_{2 \pi}^{u}$ and $R_{2 \pi}^{s}$. Given the good agreement between $R_{\pi \pi}$ and $R_{K^{+} K^{-}}$documented in Fig. 3, we restrict our analysis to the data for $K^{+} K^{-}, K^{0} \frac{\pi}{K^{0}}$, and $\eta \pi^{0}$ production from now on. First we perform an analysis for each individual value 1 of $s$ where the $K^{0} \bar{K}^{0}$ cross section is measured by BELLE [7]. We extract the quark-level form factors from the relations in (23), integrated over $\theta$ as specified in Sect. 11. The results of this procedure (henceforth referred to as single-energy analysis) are listed in Tab. 1 and displayed in the left panel of Fig. 4. While $R_{2 \pi}^{u}$ is quite well determined, $R_{2 \pi}^{s}$ suffers from rather large uncertainties. Nevertheless, a tendency for decreasing values of the scaled form factors can be seen in the plot. Particular noteworthy is the behavior of the relative phase. As shown in the right panel of Fig. 4, the phase is strongly energy dependent and seems to tend towards $180^{\circ}$. In other words, the two form factors are found to be essentially opposite in sign at the high-energy end of the data.

A remark concerning the data at $s=14.44 \mathrm{GeV}^{2}$ is in order. It turns out that at this energy the square of the non-valence form factor is negative if the central values of the cross sections are inserted into (23). There is, however, no inconsistency if the experimental errors

\footnotetext{
${ }^{1}$ At $s=14.44 \mathrm{GeV}^{2}$ no results are given for the $K^{+} K^{-}$and $\eta \pi^{0}$ channels, and we interpolate by taking the weighted average of cross sections at the two adjacent energies.
} 


\begin{tabular}{|c||c|c|c|}
\hline$s\left[\mathrm{GeV}^{2}\right]$ & $\cos \rho$ & $s\left|R^{u}\right|\left[\mathrm{GeV}^{2}\right]$ & $s\left|R^{s}\right|\left[\mathrm{GeV}^{2}\right]$ \\
\hline 8.70 & $-0.73 \pm 0.08$ & $1.32 \pm 0.07$ & $0.48 \pm 0.17$ \\
9.30 & $-0.81 \pm 0.05$ & $1.37 \pm 0.08$ & $0.60 \pm 0.16$ \\
9.92 & $-0.89 \pm 0.13$ & $1.32 \pm 0.08$ & $0.45 \pm 0.20$ \\
10.56 & $-1.02 \pm 0.44$ & $1.41 \pm 0.09$ & $0.36 \pm 0.28$ \\
14.44 & -1.00 & $1.16 \pm 0.12$ & $0.36 \pm 0.33$ \\
14.44 & $-1.49 \pm 3.80$ & $1.16 \pm 0.12$ & $0.19 \pm 0.63$ \\
\hline
\end{tabular}

Table 1: Single-energy analysis of the data for $K^{+} K^{-}, K^{0} \bar{K}^{0}$, and $\eta \pi^{0}$. Mixing between $\eta$ and $\eta^{\prime}$ is ignored. The difference between the two entries for $s=14.44 \mathrm{GeV}^{2}$ is explained in the text.

are taken into account. Therefore, we determine the quark-level form factors in the sense of a $\chi^{2}$-fit at this energy. We utilize two variants of the fit: in the first version we fix $\cos \rho$ at -1 and fit only the absolute values of the two form factors. The corresponding result is given in the penultimate row of Tab. 1, and has $\chi_{\min }^{2}=0.55$ for three data points and two free parameters. In the second version of the fit, we allow $\cos \rho$ to take any value. This gives $\chi_{\min }^{2}=0.12$ and the values listed in the last row of the table. The errors on $\cos \rho$ and $\left|R^{s}\right|$ are extremely large in this case. The difficulties to determine the quark-level form factors at $s=14.44 \mathrm{GeV}^{2}$ reflect the violation of the lower bound for $R_{\eta \pi^{0}}$ by the central values of the cross section, which we discussed at the end of the previous section.

So far we have neglected $\eta-\eta^{\prime}$ mixing in our analysis, i.e. we have approximated the $\eta$ as a pure flavor octet meson $\eta_{8}$. To assess the uncertainties introduced by this approximation, we repeat our single-energy analysis with an estimate of mixing effects. For simplicity (and lack of better information) we assume that the amplitude for annihilation into a pair of flavor-singlet mesons, $\gamma \gamma \rightarrow \eta_{1} \eta_{1}$, is negligible. We then only need to multiply the $\eta \pi^{0}$ cross section obtained in the pure octet approximation by $\cos ^{2} \theta_{P S}$. For the pseudoscalar mixing angle $\theta_{P S}$ we take the value $15.4^{\circ}$ from [13], which corresponds to $\cos ^{2} \theta_{P S}=0.93$. We find that this changes the results only marginally, the differences being well within the errors quoted in Tab. 1.

In order to reduce errors and to exploit the full set of experimental data, we now perform a combined energy-dependent fit to the three channels $K^{+} K^{-}, K^{0} \bar{K}^{0}$, and $\eta \pi^{0}$. For this fit we parameterize the form factors as

$$
s\left|R_{2 \pi}^{u}\right|=a_{u}\left(\frac{s_{0}}{s}\right)^{n_{u}}, \quad s\left|R_{2 \pi}^{s}\right|=a_{s}\left(\frac{s_{0}}{s}\right)^{n_{s}},
$$

where we chose $s_{0}=9 \mathrm{GeV}^{2}$. Guided by the results listed in Tab. 1 we take the following parameterization of the relative phase:

$$
\rho=\pi\left[1+\tanh \frac{\kappa}{s-s_{c}}\right],
$$



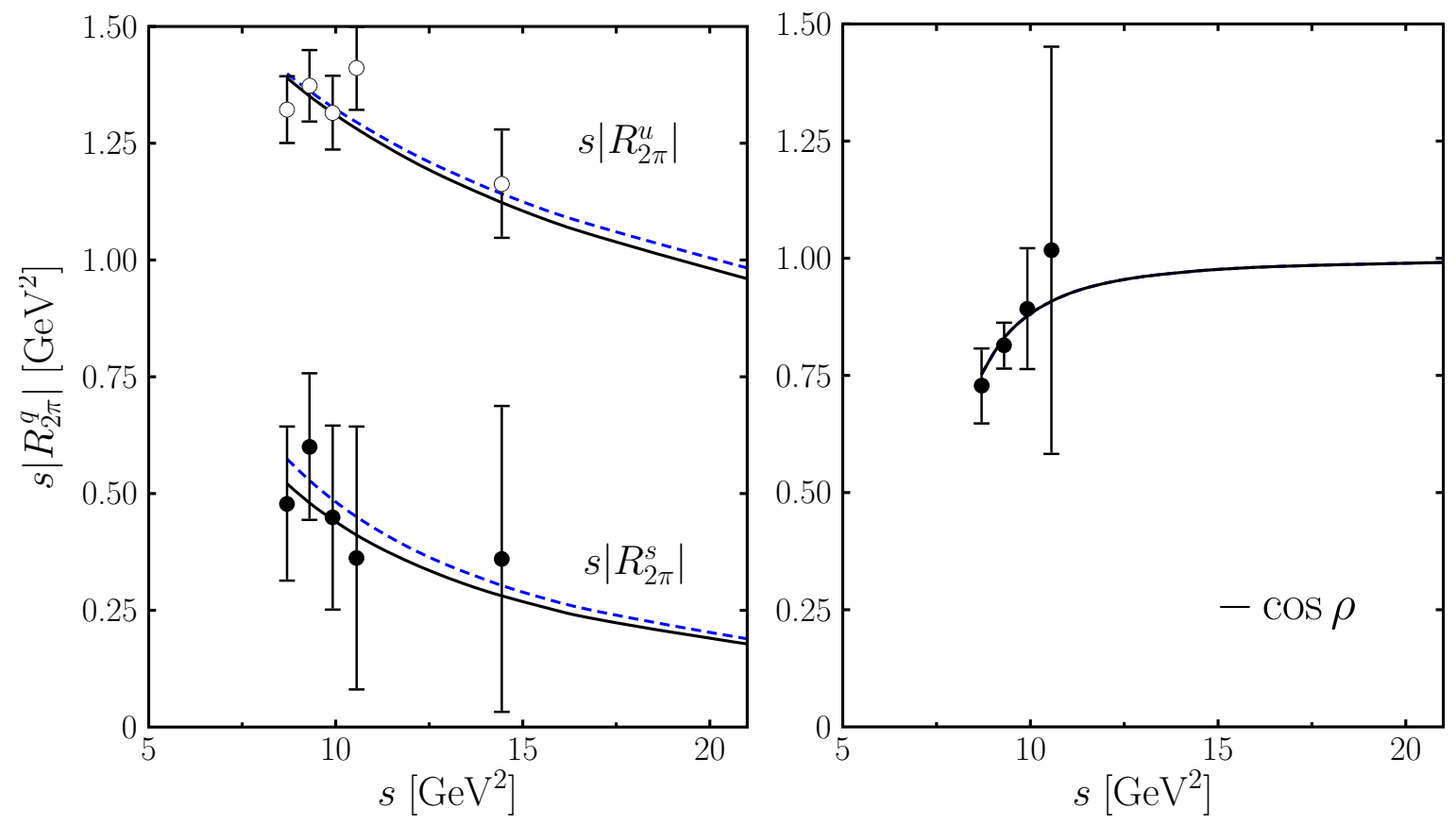

Figure 4: The scaled quark-level form factors $s\left|R_{2 \pi}^{u}\right|$ and $s\left|R_{2 \pi}^{s}\right|$ (left) as well as the cosine of their relative phase $\rho$ (right). The data points are the results of the single-energy analysis described in the text. The solid and dashed curves have the same meaning as in Fig. 2,

where $\kappa$ is constant to be adjusted to the data. For $s \rightarrow \infty$ we have $\rho \rightarrow \pi$. The constant $s_{c}$ is introduced in order to have a strong variation of $\cos \rho$ in the range of $s$ between 8.7 and $14.44 \mathrm{GeV}^{2}$, as is suggested by the results of the single-energy analysis (see Fig. 4). Our default choice for this parameter is $s_{c}=6.0 \mathrm{GeV}^{2}$, and as alternatives we take $s_{c}=5$ and $7 \mathrm{GeV}^{2}$. We also perform a fit with an estimate of $\eta-\eta^{\prime}$ mixing, assuming again that $\eta_{1} \eta_{1}$ production is negligible. We fit all data with $s \geq 8.7 \mathrm{GeV}^{2}$ and give the resulting parameters in Tab. 2. With 28 data points altogether, the minimum $\chi^{2}$ per degree of freedom is very low for all fits. We should recall in this context that we have included both the statistical and systematic errors of the data when evaluating $\chi^{2}$. As some of the systematic uncertainties are not uncorrelated between the data points, a more refined analysis would give larger values of $\chi_{\min }^{2}$ than those in our table.

In Fig. 2 we compare the process form factors evaluated from fits 1 and 2 with those we directly extracted from the BELLE data. The results of the two fits practically coincide and agree well with the data. We also compare the fit results with the quark-level form factors we derived in our single-energy analysis. Again good agreement is obtained, as shown in Fig. 4 . We observe that the fitted form factors (32) do not respect dimensional counting, and that the non-valence form factor falls off more rapidly with energy, so that at large energies the valence form factor is dominant. As discussed in the previous section, this is fully consistent with the soft handbag picture. However, due to the weight factors of $R_{2 \pi}^{u}$ and $R_{2 \pi}^{s}$ in (22), the approach to valence dominance is very slow for $R_{K^{0} \bar{K}^{0}}$. At $s=100 \mathrm{GeV}^{2}$, for instance, the valence dominance relation (29) still receives corrections of about $10 \%$ when evaluated with 


\begin{tabular}{|c|c|c||l|l|l|l|l|}
\hline fit & $s_{c}\left[\mathrm{GeV}^{2}\right]$ & $\chi_{\min }^{2}$ & $a_{u}\left[\mathrm{GeV}^{2}\right]$ & \multicolumn{1}{|c|}{$n_{u}$} & \multicolumn{1}{|c|}{$a_{s}\left[\mathrm{GeV}^{2}\right]$} & \multicolumn{1}{|c|}{$n_{s}$} & $\kappa\left[\mathrm{GeV}^{2}\right]$ \\
\hline 1 & 6.0 & 14.6 & $1.37 \pm 0.03$ & $0.42 \pm 0.08$ & $0.50 \pm 0.05$ & $1.22 \pm 0.40$ & $0.63 \pm 0.06$ \\
2 & 6.0 & 13.8 & 1.38 & 0.40 & 0.55 & 1.26 & 0.63 \\
3 & 7.0 & 14.8 & 1.38 & 0.43 & 0.46 & 1.09 & 0.40 \\
4 & 5.0 & 14.9 & 1.37 & 0.42 & 0.50 & 1.21 & 0.84 \\
\hline
\end{tabular}

Table 2: Energy-dependent fits to the $K^{+} K^{-}, K^{0} \bar{K}^{0}$, and $\eta \pi^{0}$ data with $s \geq 8.7 \mathrm{GeV}^{2}$. Fit 2 includes an estimate of $\eta-\eta^{\prime}$ mixing as explained in the text, whereas fits 1,3 , and 4 neglect mixing. The errors on the parameters in fits 2 to 4 are very similar to those in fit 1 and not listed for better legibility.

our fitted form factors. In contrast to $R_{K^{0}} \bar{K}^{0}$, the form factor $R_{K^{+} K^{-}}$is strongly dominated by the valence contribution. This provides some justification for the neglect of the non-valence form factor in our previous analysis [1], where we had only data for the $\pi^{+} \pi^{-}$and $K^{+} K^{-}$ channels at our disposal.

Figure 5 finally shows the integrated cross sections for $\eta \pi^{0}, \eta \eta$, and $K_{S} K_{S}$ production resulting from our fits. The $\eta \eta$ cross section has not been measured as yet. We also estimate it directly from the $K^{+} K^{-}, K^{0} \bar{K}^{0}$, and $\eta \pi^{0}$ cross sections using the $\mathrm{SU}(3)$ relation (15), and we find good agreement with the fit results. In contrast to all other quantities discussed so far, $\eta-\eta^{\prime}$ mixing is of greater importance for the $\eta \eta$ cross section, which is multiplied by $\cos ^{4} \theta_{P S} \approx 0.86$ in fit 2 . The difference between fits 1 and 2 can be considered as the uncertainty of our prediction for this cross section due to $\eta-\eta^{\prime}$ mixing.

The ALEPH collaboration has also measured the cross sections for two-photon annihilation into $\pi^{+} \pi^{-}$and $K^{+} K^{-}[10]$. The errors of these data are, however, so large that they have no influence if we include them in our fits. Of some interest are the two data points for the $\pi^{+} \pi^{-}$ channel at energies larger than those of the BELLE results. From these data we obtain

$$
\begin{aligned}
s\left|R_{\pi \pi}\right| & =0.84 \pm 0.42 \mathrm{GeV}^{2} & \text { at } s & =18.1 \mathrm{GeV}^{2}, \\
& =0.80 \pm 0.34 \mathrm{GeV}^{2} & & =27.6 \mathrm{GeV}^{2}
\end{aligned}
$$

when neglecting a possible $I=2$ contribution, which we expect to be small at these high energies. The values of the form factor from our fit 1 are $s\left|R_{\pi \pi}\right|=0.59 \mathrm{GeV}^{2}$ at $s=18.1 \mathrm{GeV}^{2}$ and $s\left|R_{\pi \pi}\right|=0.48 \mathrm{GeV}^{2}$ at $s=27.6 \mathrm{GeV}^{2}$, in fair agreement with the ALEPH data.

\section{Some remarks on the hard-scattering mechanism}

In this section we point out some differences between the soft handbag approach and the hardscattering mechanism of Brodsky and Lepage [3]. Given the focus of the present work, we emphasize the relations between different meson channels in the two mechanisms.

The nonperturbative quantities required in the hard-scattering picture are the leading-twist meson distribution amplitudes, characterized by the meson decay constants and the normalized 


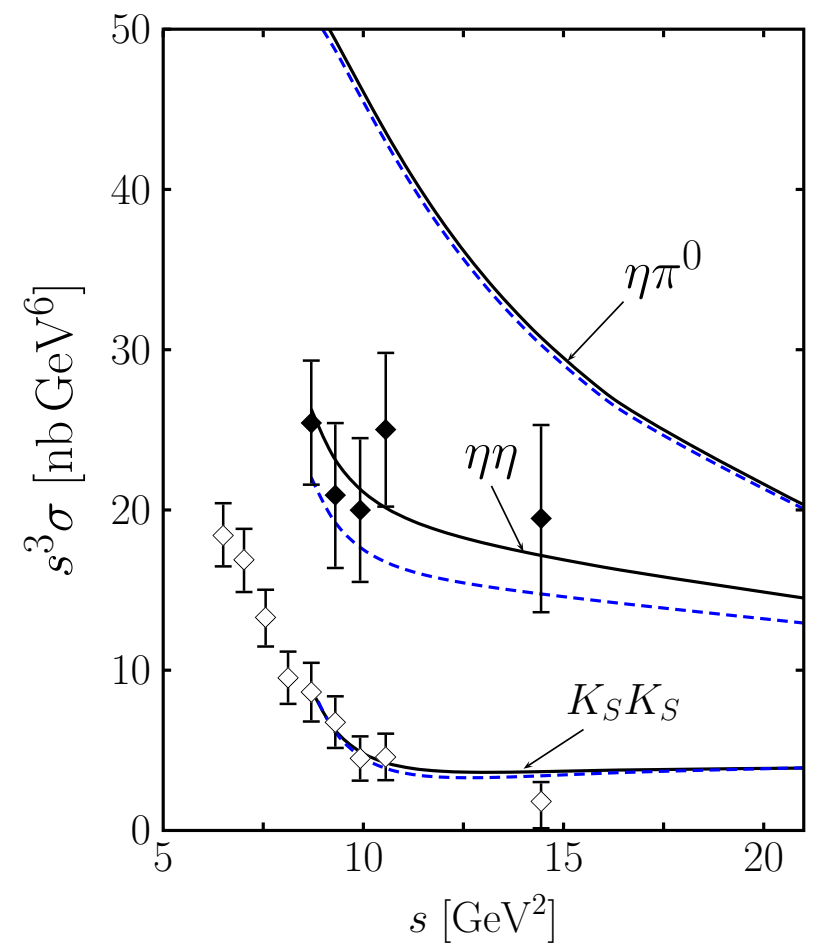

Figure 5: Predictions for the $\eta \eta$ cross section, integrated over $-0.6<\cos \theta<0.6$ and scaled by $s^{3}$. For comparison we also show the corresponding $\eta \pi^{0}$ and the $K_{S} K_{S}$ cross sections, with the kaon data (open diamonds) taken from [7]. The filled diamonds represent the $\eta \eta$ cross section evaluated from the BELLE data [5, 7, 8] with the relation (15). The solid (dashed) curves represent the cross sections obtained from fit 1 (2) in Tab. 2, note that they do not include contributions from charmonium decays.

distribution amplitudes $\phi_{M}(z)$. The asymptotic shape $\phi_{M}(z)=6 z(1-z)$ has been used for many channels in [4] and for $\pi^{+} \pi^{-}$and $K^{+} K^{-}$in [14], where the hard-scattering subprocess was evaluated at next-to-leading order in $\alpha_{s}$. The relation between the cross sections for different channels is then governed by charge factors and by the meson decay constants, which are well known for the pseudoscalar octet. In particular the ratio of $K^{+} K^{-}$and $\pi^{+} \pi^{-}$cross sections is given by $\left(f_{K} / f_{\pi}\right)^{4} \approx 2.0$ in this case, with $f_{K} / f_{\pi}$ from [15]. Such a strong breaking of the $\mathrm{SU}(3)$ symmetry relation (6) is ruled out by the BELLE data [5] for energies up to $s=16.4 \mathrm{GeV}^{2}$. We note that the absolute size of cross sections in the above calculations is significantly below the data [14]. This also holds in the modified hard-scattering approach, where Sudakov resummation and intrinsic transverse momentum of the quarks in the mesons are taken into account [16].

At moderately large scales it is natural to expect that $\phi_{M}(z)$ deviates from its asymptotic form under evolution, and one can thus have SU(3) symmetry breaking in the shapes of the distribution amplitudes. This was investigated in [17, 18] using QCD sum rule estimates. With these estimates, the $\mathrm{SU}(3)$ breaking effects in the shapes of distribution amplitudes largely compensate those in the meson decay constants. A ratio of $\pi^{+} \pi^{-}$and $K^{+} K^{-}$cross sections close to unity was obtained, in agreement with the BELLE data. 
The production of neutral meson pairs in the hard-scattering mechanism is generically suppressed, since it turns out that at leading order in $\alpha_{s}$ the bulk of the amplitude is proportional to a charge factor $\left(e_{q_{1}}-e_{q_{2}}\right)^{2}$ for a meson with quark content $q_{1} \bar{q}_{2}$ [4]. The explicit calculations in [4] and in [17, 18] yield values below 0.05 for the ratio $R$ of integrated $\pi^{0} \pi^{0}$ and $\pi^{+} \pi^{-}$ cross sections. According to (25) this requires $I=0$ and $I=2$ transitions of comparable size, in sharp contrast to the situation in the handbag approach. The values of $R$ obtained in [4, 17, 18] are significantly below the experimental results (shown in our Fig. 1). Because of charge factors, the ratio of $K_{S} K_{S}$ and $K^{+} K^{-}$cross sections in [17, 18] is even smaller, and it was concluded in [18] that at BELLE energies the $K_{S} K_{S}$ channel is dominated by contributions other than the hard-scattering one.

As for the $\eta \eta$ channel, our prediction for the $\eta \eta$ cross section differs markedly from the one obtained with asymptotic distribution amplitudes in [4, where the ratio of $\eta \eta$ and $\pi^{0} \pi^{0}$ cross sections is given by the factor $0.4\left(f_{\eta} / f_{\pi}\right)^{4}$. If one ignores $\eta-\eta^{\prime}$ mixing and takes for the ratio of $\eta$ and $\pi$ decay constants the value 1.28 from [13], one obtains a cross section ratio of about 1.1, whereas our fit 1 in the handbag approach gives about 0.3 .

We conclude this section with the remark that the new BaBar measurement [19] of the $\pi \gamma$ transition form factor at high momentum transfer is challenging our knowledge about the shape of the pion distribution amplitude. In this light one may expect that numerical estimates for $\gamma \gamma \rightarrow M \bar{M}$ within the hard-scattering picture will need to be revised.

\section{Summary}

Combined with SU(3) flavor symmetry, the soft handbag approach for two-photon annihilation into pairs of pseudoscalar mesons provides a good description of the experimental data for $s \gtrsim 9 \mathrm{GeV}^{2}$, which have been obtained by the BELLE collaboration in the recent years. The measured ratio of $\pi^{0} \pi^{0}$ and $\pi^{+} \pi^{-}$production rates implies the presence of an isospin $I=2$ contribution at $s \sim 9 \mathrm{GeV}^{2}$, which cannot be generated by the handbag mechanism. Depending on its relative phase, this contribution can, however, be as small as $10 \%$ at the amplitude level, which we regard as a quite tolerable correction to the handbag approach at this energy.

Flavor symmetry allows one to express the annihilation form factors for all processes in terms of only two quark-level form factors, one for valence quarks and a second one for nonvalence quarks. We have determined these form factors in two ways, first in a single-energy analysis and then in energy-dependent fits, with good agreement between the two methods. In our energy-dependent fits we find that the non-valence form factor is suppressed by nearly a power of $s$ compared to the valence one. This is in agreement with the physics of the handbag, which requires that the quark or antiquark entering the meson take most of its momentum. We find that the relative phase between the two form factors tends to $180^{\circ}$ at the highest energies $\left(s \sim 16 \mathrm{GeV}^{2}\right)$ of the data.

The success of our analysis implies that the present data do not provide evidence for $\mathrm{SU}(3)$ flavor violation in the annihilation form factors at the level of the experimental errors, which are typically less than $10-15 \%$. The ultimate confirmation of this picture would be an experimental verification of our prediction for the $\gamma \gamma \rightarrow \eta \eta$ cross section. 


\section{Acknowledgments}

We gratefully acknowledge correspondence with S. Uehara. This work is supported in part by BMBF, contract no. 06RY258, and by the European Project Hadron Physic 2IA in EU FP7.

\section{References}

[1] M. Diehl, P. Kroll and C. Vogt, Phys. Lett. B 532 (2002) 99 arXiv:hep-ph/0112274.

[2] A. V. Radyushkin, Phys. Rev. D 58 (1998) 114008 |arXiv:hep-ph/9803316];

M. Diehl, Th. Feldmann, R. Jakob and P. Kroll, Eur. Phys. J. C 8 (1999) 409 arXiv:hep-ph/9811253.

[3] G. P. Lepage and S. J. Brodsky, Phys. Rev. D 22 (1980) 2157.

[4] S. J. Brodsky and G. P. Lepage, Phys. Rev. D 24 (1981) 1808.

[5] H. Nakazawa et al. [BELLE Collaboration], Phys. Lett. B 615 (2005) 39 arXiv:hep-ex/0412058.

[6] S. Uehara et al. [BELLE Collaboration], Phys. Rev. D 79 (2009) 052009 arXiv:0903.3697 [hep-ex]].

[7] W. T. Chen et al., Phys. Lett. B 651 (2007) 15 arXiv:hep-ex/0609042.

[8] S. Uehara et al. [BELLE Collaboration], Phys. Rev. D 80 (2009) 032001 arXiv:0906.1464 [hep-ex]].

[9] J. Dominick et al. [CLEO Collaboration], Phys. Rev. D 50 (1994) 3027 arXiv:hep-ph/9403379.

[10] A. Heister et al. [ALEPH Collaboration], Phys. Lett. B 569 (2003) 140.

[11] G. W. London, Fortsch. Phys. 12 (1964) 643.

[12] M. Diehl, P. Kroll and C. Vogt, Eur. Phys. J. C 26 (2003) 567 arXiv:hep-ph/0206288.

[13] Th. Feldmann, P. Kroll and B. Stech, Phys. Rev. D 58 (1998) 114006 arXiv:hep-ph/9802409.

[14] G. Duplančić and B. Nižić, Phys. Rev. Lett. 97 (2006) 142003 arXiv:hep-ph/0607069].

[15] C. Amsler et al. [Particle Data Group], Phys. Lett. B 667 (2008) 1.

[16] C. Vogt, AIP Conf. Proc. 571 (2001) 345 arXiv:hep-ph/0010040.

[17] M. Benayoun and V. L. Chernyak, Nucl. Phys. B 329 (1990) 285.

[18] V. L. Chernyak, Phys. Lett. B 640 (2006) 246 arXiv:hep-ph/0605072.

[19] B. Aubert et al. [The BaBar Collaboration], Phys. Rev. D 80 (2009) 052002 arXiv:0905.4778 [hep-ex]]. 